\title{
Integrated approach for optimal island partition and power dispatch
}

\author{
Junpeng ZHU ${ }^{1}$, Wei GU ${ }^{1} \oplus$, Ping JIANG ${ }^{1}$, Zhi WU ${ }^{1}$, \\ Xiaodong YUAN ${ }^{2}$, Yinghui NIE ${ }^{1}$
}

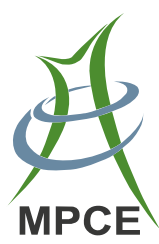

\begin{abstract}
Operating in island mode when failure occurs in the active distribution network(ADN) is an effective measure to maintain an uninterrupted power supply to significant loads. A two-stage approach that integrates optimal island partition and power dispatch is proposed in this paper, considering photovoltaics (PVs), batteries (BEs) and electric vehicles (EVs) as the power sources. In the first stage, energy indices are defined to describe the energy demand and the maximum energy that these distributed energy resources (DERs) can provide, and islands are partitioned based on an energy constraint. Considering the variability the loads and PVs, the energy constraint is a necessary but not sufficient condition for island operation, so in the second stage, a power dispatch model is proposed
\end{abstract}

CrossCheck date: 4 May 2017

Received: 23 July 2016/Accepted: 4 May 2017/Published online: 1 November 2017

(C) The Author(s) 2017. This article is an open access publication

$\triangle$ Wei GU

wgu@seu.edu.cn

Junpeng ZHU

zhujp_0705@163.com

Ping JIANG

jping@seu.edu.cn

Zhi WU

zwu@ seu.edu.cn

Xiaodong YUAN

lannyyuan@hotmail.com

Yinghui NIE

nyhnieyinghui@qq.com

1 School of Electrical Engineering, Southeast University, Nanjing, China

2 Jiangsu Electric Power Company Research Institute, Nanjing, China as a test for the island partition result. Sequential power flow is also simulated to guarantee a feasible and optimized island status. The situations when the tests are not passed are analyzed and classified, and corresponding modifications for the first stage model are provided. Multiple levels of constraints based on the energy index are established for the island partition model. The proposed approach has been validated through simulation using a modified IEEE 69-bus system which is divided into three districts with different load variability characteristics.

Keywords Optimal island partition, Distributed generation, Island power dispatch, Active distribution networks, Photovoltaic, Electric vehicles

\section{Introduction}

Conventionally, power is supplied to electrical customers via large central power plants through transmission and distribution networks. With distributed energy resources (DERs) accessing it in high density, the distribution network transforms from passive to active with bi-directional flow. According to the definition of CIGRE [1], an active distribution network (ADN) utilizes DERs and flexible grid topology to manage power flows. It has been estimated that $80 \%$ of the supply interruptions faced by customers are because of failures that occur in the distribution network. In an ADN, the loads no longer have only a single power supply path because of the introduction of DERs, such as distributed generation (DG), batteries (BEs), and plugged-in electric vehicles (EVs). The increasing penetration levels of DERs together with suitable control strategies are making it possible for an ADN to meet higher reliability requirements and improve power supply security 
[2-4]. When the network undergoes some disturbance, one promising strategy is to operate some portions of the ADN in island mode to ensure uninterrupted power supplies for some of the significant loads [5-7]. In this situation, the island partition and the power dispatch method within islands are the most important variables for maximizing the benefits of the available DERs.

Islanding and islanded operation have been widely investigated by many researchers. In terms of optimal island partition, a systematic approach considering system reliability and supply security for optimal microgrid design was presented in [8]. The optimal design considers both sustained and temporary faults to determine system reliability via a combined probabilistic reliability index, and considers the real and reactive power balances for supply security. However, the microgrids in this approach are not islanded to deal with failures, but rather for long-term operation. An multi-objective model for optimal island partition after fault isolation is proposed in [9]. Maximization of the amount of priority loads and the total loads, minimization of the number of new switches to be installed and the switching operations are considered as the objectives in this approach. An island partition algorithm that considers both the real and reactive power balances is proposed in [10]. A highly efficient multilevel multi-objective graph partitioning technique is applied so that the model is applicable to very large power grids. In [11-14], the optimal single-DG island partition is formulated as a tree knapsack problem (TKP). The TKP model is solved using a branch and bound algorithm in [11], an intelligent algorithm in [12], a heuristic searching algorithm in [13], and a modified shuffled frog leap algorithm (SFLA) in [14]. However, in [9-14], the island partition models are all described as static, i.e., the loads and the outputs of the DGs are all expected to be constant during the island partition period.

In terms of optimal power dispatch for the islands (microgrids), the variability of loads and DGs during the partition period are commonly considered [15-18]. In [15], the DGs are separated into two ownership groups-utilities and independent power producers - and only the utilityowned DGs with battery energy storage systems are expected to be controllable. Functionality to manage island storage considering the integration of EVs and responsive loads to achieve power balance are proposed in [16]. In $[17,18]$, stable operation in islanded mode is considered as a constraint during grid-connected operation. The objective of islanded operation in [17] is to minimize power mismatches, while [18] pays more attention to power flow limits. The economic dispatch model is applied for gridconnected operation in both $[17,18]$. Both island partition and the static power dispatch are considered in [19]. However, the static power dispatch of DERs is independent of the island partition process, and is done when the island scope has been determined. The variability of the loads and the DERs are not considered in [19].

Existing island partition methods commonly deal with static situations, in which the loads and the outputs of the DERs are set to be constant, while variability of both loads and DERs must be considered in islanded operation. To address this issue, this paper proposes an integrated twostage approach, introducing an energy index to maintain the partition part as static, and using DER power dispatch and power flow simulation to calculate the output schedules of the DERs, test the power balance and modify the partition model. The contributions of this approach are as follows.

1) The energy index is first introduced to the island partition model. Previous work has considered power balance to be the major constraint for island partition, but static power can only describe the situation at a specific time interval. The energy index contains information about the variability of DGs and loads throughout the entire partition period, and the model remains in TKP form and is thus solvable.

2) The power dispatch process and sequential power flow simulation are considered to test the partition status and modify the partition model. A constraint set containing multi-level constraints which are applicable for different scenarios is established for the island partition process to guarantee a feasible and optimized partition.

The remainder of this paper is organized as follows. The framework of the two-stage model is given in Section 2. Definitions of the energy index and the island partition model considering the energy constraint are described in Section 3. The DER dispatch and power flow simulation are proposed in Section 4. Modification method and adjustment strategies for the island partition model are proposed in Section 5, and case simulations are presented in Section 6. Finally, Section 7 gives the conclusions for this paper.

\section{Framework of the two-stage model}

The framework of the two-stage model is shown in Fig. 1.

In the first stage, the energy indices of loads, DERs (including DGs, BEs, and EV integrations), and nodes in the distribution network are firstly defined, based on which the energy constraints can be expressed. The energy constraints and radial structure constraints constitute the initial constraint set of the island partition model. 


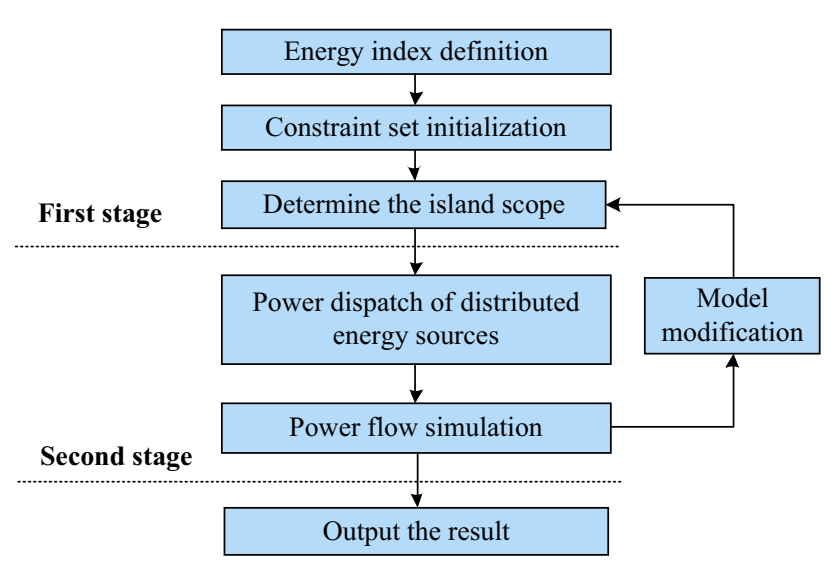

Fig. 1 Framework of the two-stage model

The island scope determined in the first stage model can ensure that the net output energy of the island is positive during the entire partition period. However, it cannot be ensured that the net output power of the island is positive for any time interval within that period, nor that the power flow constraints and security limit constraints are met. So in the second stage, two steps ensure the feasibility of the islands:

Step 1: For the island determined in the first stage, a DER power dispatch model is proposed to check whether the net output power of the island can be kept positive in each time interval.

Step 2: Based on the DER output schedule determined in Step 1, a sequential power flow simulation is performed to check whether the security limit constraints can be satisfied and to account for power losses.

Modification of the island partition model is proposed based on the results of Steps 1 and 2. Power constraint or energy transfer constraint will be added to the constraint set of the island partition model if the test in Step 1 is not passed. Adjustment strategies such as voltage regulation of DGs and load shedding will be used if the test in Step 2 is not passed.

\section{First stage: optimal island partition}

The combination of PV generation and a BE system, called a PV/BE station, is considered as the primary energy resource for the island power supply in this paper. An example distribution network with $\mathrm{PV} / \mathrm{BE}$ stations, EV connection points, and other load points is shown in Fig. 2. Under grid-connected conditions, the PV generation is always controlled to operate with maximum power point tracking (MPPT) for maximum economic benefit. When failure occurs in the network and the PV/BE stations are switched to island operation mode, the BE systems adopt a $V / f$ control strategy to ensure a stable frequency in the

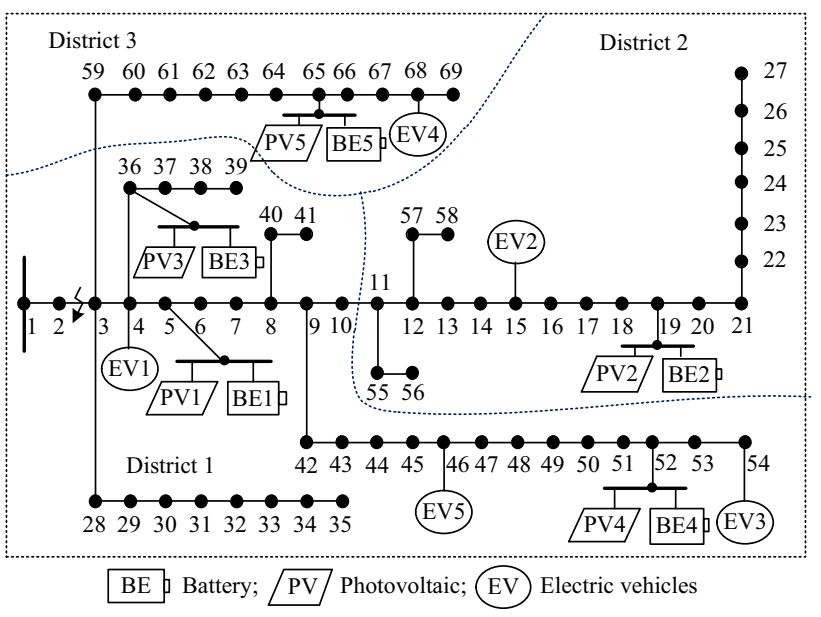

Fig. 2 Distribution network with PV/BE stations and EVs

island and relieve the power imbalance between the loads and the PV generation output [20]. Therefore, each PV/BE station is equipped with suitable power conversion and control equipment to provide these services. EV integration with a vehicle-to-grid (V2G) facility is also considered as an energy resource for the island, but without enough frequency regulation capability to support the island unaided.

\subsection{Definition of the energy indices}

Energy indices are defined at each network node. The energy index of the load describes the energy demand during the island partition period, and the energy indices of the DERs describe the maximum energy that they can provide for the island The energy index expressions for BEs, loads, PV generation, and EV integration are defined in (1)-(4), respectively.

$E_{B E, i}=S_{O C C_{B E, i}}\left(T_{s}\right) \cdot C_{B E, i}$

$E_{L, i}=-\int_{T_{s}}^{T_{e}} P_{L, i}(t) \mathrm{d} t$

$E_{P V, i}=\int_{T_{s}}^{T_{e}} P_{P V, i}(t) \mathrm{d} t$

$E_{E V, i}=\sum_{j=1}^{N_{E V, i}} \operatorname{SOC}_{E V, i, j}\left(T_{s}\right) \cdot C_{E V}$

where $T_{s}$ and $T_{e}$ are the start time and the end time of the island partition period; $S O C_{B E, i}$ is the state of charge of the $\mathrm{BE}$ at node $i, S_{E O C_{E V, i j}}$ is the state of charge of the $j^{\text {th }} \mathrm{EV}$ connected at node $i, C_{B E, i}$ and $C_{E V}$ are the energy capacities of the BE at node $i$ and a single EV, $P_{L, i}(\mathrm{t})$ and $P_{P V, i}(\mathrm{t})$ are the powers of the load and the PV generation at node $i$ at time $t$, and $N_{E V, i}$ is the number of EVs connected at node $i$. 
The energy index of a node is defined as the maximum net output energy of the node during the partition period:

$E_{i}=E_{B E, i}+E_{L, i}+E_{P V, i}+E_{E V, i}$

\subsection{Island partition for a single PV/BE station}

The island partition based on the energy constraint for a single $\mathrm{PV} / \mathrm{BE}$ station is described here in the form of a TKP:

$\max \sum_{i=0}^{n} x_{i} d_{i} E_{L, i}$

s.t.

$\sum_{i=0}^{n} x_{i} E_{i} \geq 0$

$x_{0}=1$

$x_{i} \in\{0,1\} \quad i=1,2, \ldots, n$

$x_{a_{i}} \geq x_{i}$

where $i$ is the node number and the PV/BE station is connected to node $0 ;\left\{x_{i}\right\}$ is the vector of control variables; $d_{i}$ is the weight of load $i$; and $a_{i}$ is the node number of the parent node of node $i$ when node 0 is considered as the root node.

If node $i$ is included in the island, $x_{i}$ is 1 . The weighted uninterrupted energy is maximized in (6). Equation (7) is the energy constraint, which ensures that the net output energy of the island is positive. It is a necessary but not sufficient condition for island operation. The node with the $\mathrm{PV} / \mathrm{BE}$ station is included in the island by fixing $x_{0}$ to be 1 in (8). Generally, the TKP is a non-linear problem because the structure constraint in (10) contains an implicit functional relationship between $a_{i}$ and $i$ [11-14], but with full information of the network structure, the structure constraint for all nodes can be given explicitly and therefore the model can be expressed as a linear programming (LP) problem [19].

\subsection{Island partition for multiple PV/BE stations}

The island partition process for multiple PV/BE stations is as follows:

Step 1: Partition the islands for each of the PV/BE stations using the formulation of (6)-(10) given in subsection 3.2. When partitioning the island for a specific $\mathrm{PV} / \mathrm{BE}$ station, the energy indices of the other DERs are set to 0 .

Step 2: If the islands have no intersections, end the process; if there is an intersection, then for all islands that contain this intersection, convert all the PV/BE stations and the nodes between these PV/BE stations into the equivalent of a single node by combining their energy indices:
$E_{E n, A}=\sum_{i \in \text { Area } A} E_{i}$

where $A$ is the area that includes the PV/BE stations and the nodes between these stations, and $E_{E n, A}$ is the energy index of the equivalent node representing that area.

Step 3: Cancel the intersecting islands and then partition the island for the equivalent PV/BE station node. Repeat Step 2 until the islands have no intersections.

\section{Second stage: DER dispatch and power flow tests}

The energy constraint for island partition is a necessary but not sufficient condition for island operation: the constraint can only ensure that energy is sufficient for the entire islanding period, but cannot ensure that the power can be balanced at any time interval during that period, and neither are security constraints considered. DER dispatch and power flow simulation are necessary to test the partition results and feed back modifications to the first-stage model.

\subsection{DER dispatch test}

The first step aims to check whether the net output power of the island can be kept positive in each time interval within the islanding period.

The DER dispatch model aims to determine the output schedule for DERs, and to check whether the power is sufficient. The PV generation is assumed to operate in MPPT mode, and the objective is to minimize the unsatisfied power demand during the island partition period:

$\min _{P_{B E}, P_{E V}} E_{u n}=\int_{T_{s}}^{T_{e}} P_{u n}(t) \mathrm{d} t$

s.t.

$P_{u n}(t)=\max \left(0, \sum_{i \in \Omega}\left(P_{L, i}(t)-P_{P V, i}(t)-P_{B E, i}(t)-P_{E V, i}(t)\right)\right)$

$-P_{B E, c h, i}^{\max } \leq P_{B E, i}(t) \leq P_{B E, d i s, i}^{\max }$

$P_{E V, i}(t)=n_{d i s}(t) P_{E V, d i s}^{\max }-n_{c h}(t) P_{E V, c h}^{\max }$

$n_{\text {dis }, i}(t) \leq r_{\text {obey }} \frac{E_{E V, i}(t)}{C_{E V}}$

$n_{c h, i}(t) \leq r_{\text {obey }}\left(N_{E V, i}-\frac{E_{E V, i}(t)}{C_{E V}}\right)$

$\sum_{i \in \Omega} n_{d i s, i}(t) \sum_{i \in \Omega} n_{c h, i}(t)=0$ 


$$
\begin{aligned}
& E_{B E, t}(t+\Delta t)=E_{B E, t}(t)-\int_{t}^{t+\Delta t} P_{B E, t}(t) \mathrm{d} t \\
& E_{E V, t}(t+\Delta t)=E_{E V, t}(t)-\int_{t}^{t+\Delta t} P_{E V, t}(t) \mathrm{d} t \\
& 0 \leq E_{B E, i}(t) \leq C_{B E, i} \\
& 0 \leq E_{E V, t}(t) \leq N_{E V, t} C_{E V}
\end{aligned}
$$

where $P_{B E}$ and $P_{E V}$ are the control variables; $P_{u n}(t)$ is the unsatisfied power demand at time $t ; P_{E V, i}(t)$ is the power of the EV integration in node $i$ which can be calculated using (15); $P_{B E, i}(t)$ is the power of the BE at node $I ; \Omega$ is the set of nodes in the island; $P \max B E, c h, i$ and $P \max B E$, dis, $i$ are the maximum charge and discharge powers of the BE at node $I$; and $P \max E V$,ch and Pmax EV,dis are the maximum charge and discharge powers of an EV, assumed without loss of generality to be the same for all EVs; $n_{c h, i}(t)$ and $n_{d i s, i}(t)$ are the numbers of charging and discharging EVs at node $i$ at time $t$, for which the upper limits can be estimated conservatively using (16) and (17), respectively, and $r_{\text {obey }}$ is the proportion of schedulable EVs, which reflects the EV users' participation in island power management; $E_{E V, i}(t)$ and $E_{B E, i}(t)$ are the energies of $\mathrm{EV}$ integration and the $\mathrm{BE}$ at node $i$ at time $t$, which can be calculated using (1), (4), (19) and (20). The time-stepping characteristics of BEs and EVs are considered in (19) and (20). Equation (18) guarantees that charging EVs and discharging EVs cannot coexist.

Equations (14), (16)-(18), (21) and (22) are the power and capacity constraints of the BEs and EVs, and are not considered in the first-stage model. In the situation where the variations of the PV power and the loads are relatively consistent, or where the maximum charge/discharge powers of the BEs and EVs are sufficiently high and the BEs have an abundant capacity, the power demand can be satisfied at any time and the objective function is equal to 0 . Otherwise, the optimization result of the objective function may be a positive number, meaning that the dispatch test has not been passed.

If there are multiple islands, then the dispatch process should be performed for each island. The time $t$ should be discretised to ensure that the model is solvable with the computing resources available (for example the time step can be set as $15 \mathrm{~min}$ when the islanding period is 5 hours).

\subsection{Sequential power flow test}

If the DER power dispatch test is passed, the net output power of the island can be kept positive for each time interval using the DER output schedule. However, the feasibility of the island is still not ensured because power losses and system security constraints are not accounted for. Therefore, a sequential power flow simulation is performed based on the DER output schedule determined by the dispatch model in subsection 4.1.

For each isolated island, the PV/BE station with the maximum output is set as the slack node, and other PV/BE stations, EV integration and loads are set as PQ nodes [11]. The sequential power flow can be simulated to check whether the security constraints can be satisfied and whether power balance can be achieved.

\section{Modification and adjustment based on the DER dispatch and power flow results}

The island partition result is not feasible if the DER dispatch test or the power flow test is not passed. Model modification and adjustment strategies corresponding to the tests are given in this section. Firstly, the cases that fail the DER dispatch test are analyzed and modification of the constraint set is proposed. Secondly, adjustment strategies based on the power flow test are given.

\subsection{Model modification based on the power dispatch test}

Compared with the energy constraint in the island partition model, the constraints in the dispatch model are enhanced by two means: the power exchanged between the $\mathrm{BEs} / \mathrm{EVs}$ and the network is constrained, and the energy stored in the BEs/EVs is also constrained. Therefore, on the premise that the net output energy of the island is positive (i.e., the constraint in (7) is satisfied), two cases will lead to the power dispatch test being failed:

Case A: limited ability for power support from the BEs and EV integration, because their maximum output powers are limited.

Case B: limited ability for energy transfer using the BEs and $\mathrm{EV}$ integration, because their input powers are limited (Case B-1) or their energy capacities are limited (Case B-2 and Case B-3).

Figure 3 shows a sketch of Case A. Line 1 shows the theoretical maximum outputs of the BEs and EVs, and Curve 2 shows the sum of the maximum outputs of the BEs, EVs and PVs in an island. The energy constraint can be satisfied if the BEs and EVs store sufficient energy at $T_{s}$, but there are certain periods $\left(\left[t_{0}, t_{1}\right]\right)$ during which the power demand cannot be met, even if the BEs and EVs discharge at maximum power and the PVs operate under MPPT conditions. In this situation, $P_{u n}(t)$ is positive during $\left[t_{0}, t_{1}\right]$ and the dispatch test cannot be passed.

Figure 4 shows sketches of Case B. In Fig. $4 a$ and b, the PV output is higher than the load during $\left[T_{s}, t_{m 1}\right]$ and is lower than the load during $\left[t_{m 1}, T_{e}\right]$. In Fig. $4 \mathrm{c}$, the PV 


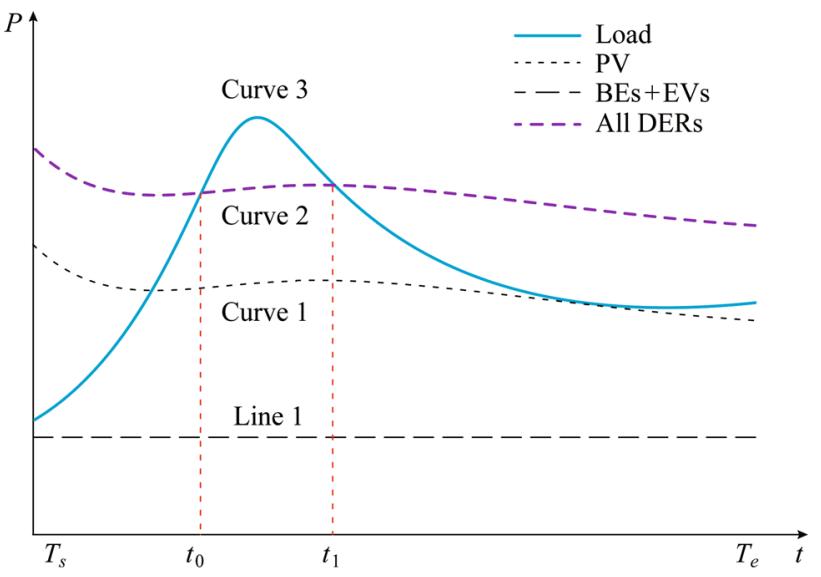

Fig. 3 Case A: limitation of the power support ability of the BEs and EVs

output is lower than the load during $\left[T_{s}, t_{m 2}\right]$ and is higher than the load during $\left[t_{m 2}, T_{e}\right]$. Suppose that the energy constraint over the entire period is satisfied.

In Case B-1 shown in Fig. 4a, the input power of the BEs and EVs is limited, and there are certain periods ([ $T_{s}$, $\left.t_{2}\right]$ ) during which the BEs and EVs cannot store the redundant power from the PVs because of charging power limitations. The wasted energy is marked as the shaded area $\mathrm{S} 1$.

In Case B-2 shown in Fig. 4b, the capacities of the BEs and EVs are limited, and there are certain periods $\left(\left[t_{4}, t_{m 1}\right]\right)$ during which the PV output is higher than the load, but the BEs and EVs cannot charge because of energy capacity limitations. In Fig. $4 \mathrm{~b}$, the BEs and EVs charge during $\left[T_{s}\right.$, $\left.t_{4}\right]$ and are all fully charged at $t_{4}$, leading to the wasted energy marked as the shaded area S2.

In Case B-3 shown in Fig. 4c, the capacity (or the SOC at time $T_{e}$ ) of the BEs/EVs is limited and the BEs/EVs are short of energy during the period $\left[t_{6}, t_{m 2}\right]$, while there are also some periods $\left(\left[t_{m 2}, T_{e}\right]\right)$ during which the energy stored by the BEs/EVs is not used by the island (because the net output energy of the island is positive), with the latter being marked as shaded area S3. This can also be regarded as a type of wasted energy.

In Cases B-1, B-2, and B-3, there are periods during which the energy is wasted and other periods ( $\left[t_{3}, T_{e}\right]$, $\left[t_{5}\right.$, $\left.T_{e}\right]$ and $\left.\left[t_{6}, t_{m 2}\right]\right)$ during which the power demand cannot be satisfied because the BEs/EVs are short of energy. In an abstract manner, it is the limitations of the ability of the BEs and EV integrations to transfer energy that lead to the dispatch test failure.

To modify the first-stage island partition model when the dispatch test is failed, we first check the case to which this situation belongs. Consider this proposition:

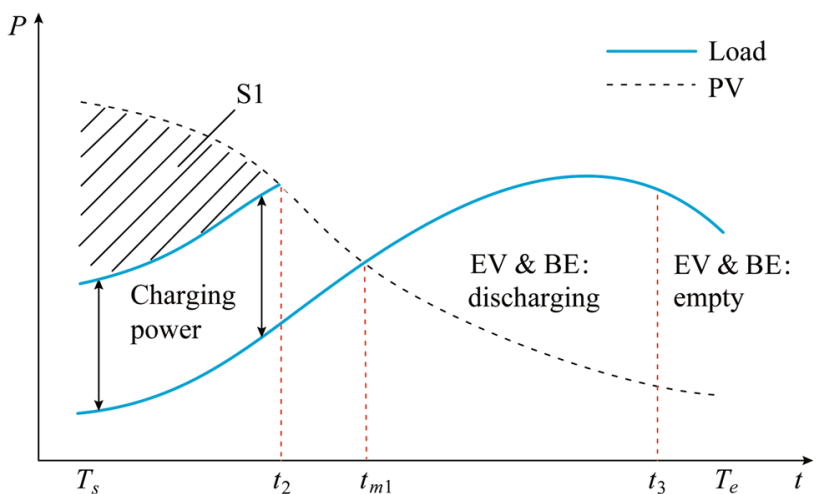

(a) Case B-1

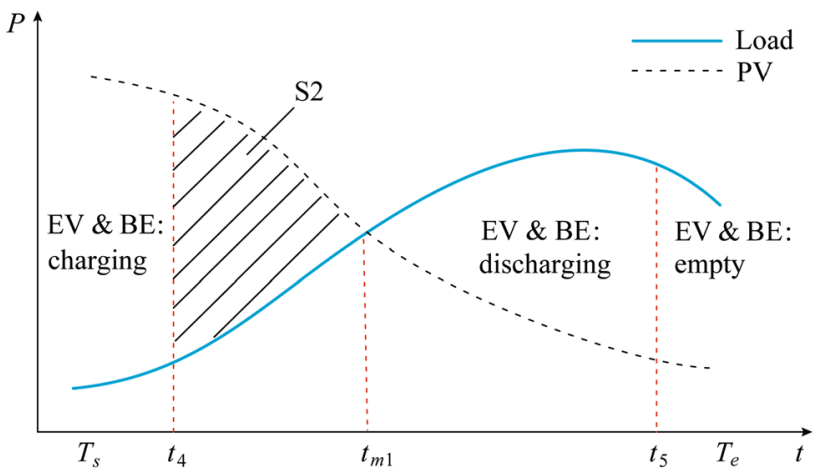

(b) Case B-2

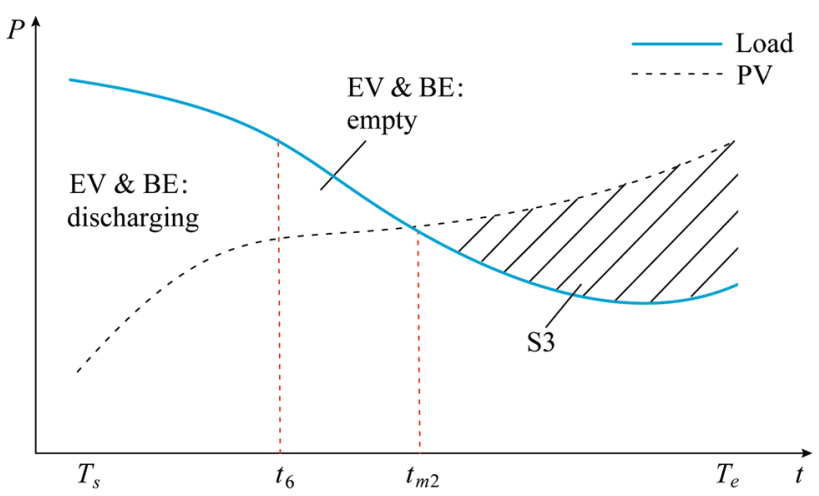

(c) Case B-3

Fig. 4 Case B: limitation of the energy transfer ability of the BEs and EVs

$\begin{cases}\exists t \in\left[t_{s}, t_{e}\right] \\ \text { s.t. } \quad P_{u n}^{\prime}=\sum_{i \in \Omega}\left(P_{L, i}(t)-P_{P V, i}(t)-P_{B E, i}^{\max }-r_{o b e y} N_{E V, i} P_{E V, d i s}^{\max }\right)>0\end{cases}$

If proposition (23) is true, we can then deduce that the situation belongs to Case A; otherwise, the situation must belong to Case $\mathrm{B}$.

For Case A, the required modification of the up-stage model is as follows: 
Step 1: According to (23), calculate the maximum $P^{\prime}{ }_{u n}(t)$ and denote by $t_{u n}$ the time when the excess of load above output of the PVs is expected to be at its greatest.

Step 2: Add the following power constraint at time $t_{u n}$ to the island partition model:

$\sum_{i=0}^{n} x_{i}\left(P_{P V, i}\left(t_{u n}\right)+P_{B E, i}^{\max }+r_{o b e y} N_{E V, i} P_{E V, d i s}^{\max }-P_{L, i}\left(t_{u n}\right)\right) \geq 0$

The constraint of (24) is still an essential but not sufficient condition for island operation, but because of the selection of $t_{u n}$ it will inevitably change the island partition result. Equations (6)-(10) and (24) constitute the modified island partition model.

If the proposition in (23) is false and the situation belongs to Case $\mathrm{B}$, then the energy transfer constraints of the BEs and EVs should be added to the island partition model, because the limitation of the ability of the BE and EV integration to transfer energy led to the dispatch test failure. First, we propose a method to estimate the energy transfer process of the $\mathrm{BE}$ and $\mathrm{EV}$ integration, as follows:

$$
\begin{aligned}
E_{B E, 0}\left(T_{s}\right) & =\operatorname{SOC}_{B E, 0}\left(T_{s}\right) C_{B E, 0} \\
E_{E V, \text { all }}\left(T_{s}\right) & =\sum_{i=0}^{n} x_{i} E_{E V, i}\left(T_{s}\right) \\
& =\sum_{i=0}^{n}\left(x_{i} \sum_{j=1}^{N_{E V, i}} \operatorname{SOC}_{E V, i, j}\left(T_{s}\right) \cdot C_{E V}\right)
\end{aligned}
$$

$E_{B E, 0}^{\prime}(T+\Delta T)=E_{B E, 0}(T)+\int_{T}^{T+\Delta T} P_{B E}(t) \mathrm{d} t$

$E_{E V, \text { all }}^{\prime}(T+\Delta T)=E_{E V, \text { all }}(T)+\int_{T}^{T+\Delta T} P_{E V, \text { all }}(t) \mathrm{d} t$

$P_{r d}(t)=P_{P V, 0}(t)-\sum_{i=0}^{i=n} x_{i} P_{L, i}(t)$

$P_{B E}(t)=\min \left(P_{B E, c h, 0}^{\max }, \max \left(P_{r d}(t),-P_{B E, d i s, 0}^{\max }\right)\right)$

$P_{E V, \text { all }}(t)=$

$$
\left\{\begin{array}{cl}
\min \left\{P_{r d}(t)-P_{B E, c h, 0}^{\max }, N_{c h, a l l} \cdot P_{E V, c h}^{\max }\right\} & P_{r d}(t) \geq P_{B E, c h, 0}^{\max } \\
0 & -P_{B E, d i s, 0}^{\max } \leq P_{r d}(t)<P_{B E, c h, 0}^{\max } \\
P_{r d}(t)+P_{B E, d i s, 0}^{\max } & P_{r d}(t)<-P_{B E, d i s, 0}^{\max }
\end{array}\right.
$$

$N_{\text {ch,all }}(t)=r_{\text {obey }}\left(\sum_{i=0}^{n} x_{i} N_{E V, i}-\frac{E_{E V, \text { all }}(t)}{C_{E V}}\right)$

$E_{B E, 0}(T+\Delta T)=\min \left(E_{B E, 0}^{\max }, E_{B E, 0}(T+\Delta T)\right)$

$E_{E V, \text { all }}(T+\Delta T)=\min \left(N_{E V, \text { all }} C_{E V}, E_{E V, \text { all }}^{\prime}(T+\Delta T)\right)$ where $\left\{x_{i}\right\}$ represents the control variables in the island partition model, and $E_{E V \text {,all }}(t)$ is the total energy of the EV integrations in the island at time $t$. Similarly to the downstage dispatch model, $N_{\text {ch,all }}$ represents the upper limit of the number of chargeable EVs that can be conservatively estimated using (32).

The time-stepping characteristics of BEs and EVs are considered in (27) and (28). According to (27)-(31), this model is an estimation method because the dispatch strategy is simplified: the imbalance between the PVs and the load is first relieved by the BEs, and then by the EVs. This strategy is a feasible approach but may not be the optimal approach.

Equations (30) and (31) consider the maximum charging power of BEs and EV integrations, and correspond to Case B-1; (33) and (34) consider the capacity limitation, and correspond to Case B-2 and Case B-3.

Based on this estimation method, we can add the energy transfer constraints of the BEs and EV integrations to the first-stage model, as follows:

$E_{B E, 0}(t) \geq 0 \quad \forall t$

$E_{E V, \text { all }}(t) \geq 0 \quad \forall t$

$N_{\text {dis,all }}(t) \geq \frac{P_{E V, \text { all }}(t)}{P_{E V, \text { dis }}^{\max }} \quad \forall t$

where $N_{\text {dis, all }}$ represents the upper limits of the number of dischargeable EVs, which can be conservatively estimated based on $E_{E V, \text { all }}$ in (38):

$N_{\text {dis,all }}(t)=r_{\text {obey }} \frac{E_{E V, \text { all }}(t)}{C_{E V}}$

Essentially, (35)-(38) represent the constraints of energy transfer but not of power. According to (29)-(31), it is supposed that the power demand can always be satisfied by following the simplified dispatch strategy, because case A has been excluded, and (35)-(38) provide the objective energy conditions required for this supposition.

Because the simplified dispatch strategy is a feasible approach but may not be the optimal approach, the modified first-stage model, including (6)-(10) and (25)-(38), contains a sufficient but not essential condition to ensure the DER dispatch test passed.

The precision of the estimation is affected by the step size $\Delta \mathrm{T}$. In the same manner as the dispatch model, time can be discretised to provide a practical and solvable model.

\subsection{Adjustment strategies based on the power flow test}

After the constraint set modification, the DER dispatch test is ensured to be passed. A sequential power flow 
simulation is then performed based on the DER output schedule determined by the dispatch model, and adjustment strategies based on power flow are available in the literature [11].

If the output power of the PV generation at the slack node is greater than its maximum output power because of network losses, some unimportant loads will be shed to ensure power balance. If some of nodes' voltage or equipment capability are beyond their limits, adjustment measures such as voltage regulation of PVs, reactive power compensation and load shedding will be taken to remove those violations. Thus, the optimal island partition scheme is implemented through a variety of measures.

\subsection{Entire flow of the integrated approach}

The entire flow of the integrated approach proposed in this paper is shown in Fig. 5.

In conclusion, three levels of constraints are sequentially applied to the island partition problem, and power flow simulation finally ensures the feasibility of the islands

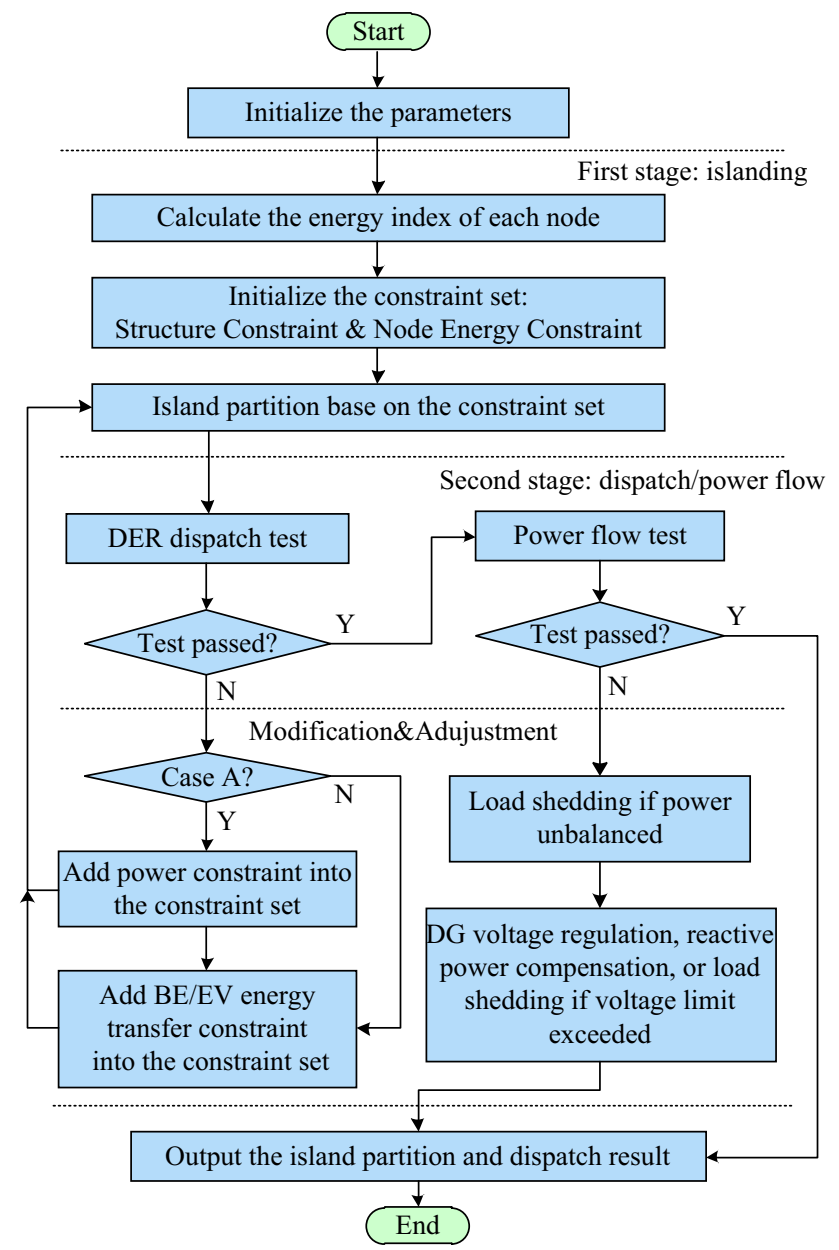

Fig. 5 Flowchart of the proposed approach partitioned. The strengths of the three constraint levels for island operation are listed as follows:

The energy constraint: essential but not sufficient, possibly leading to Case A or Case B.

The energy and power constraint: essential but not sufficient. However, this constraint can eliminate Case A.

The energy transfer constraint: sufficient to ensure that the net output power of the island is positive for any time interval within the islanding period.

\section{Test case simulation}

\subsection{Parameter settings}

A modified test feeder based on the IEEE 69-bus test system was used to test the proposed method. The PVs, the BEs and the EV charging/discharging stations are integrated into the system, as shown in Fig. 1.

The island partition period is set to be 11:00 AM to 4:00 PM. For dispatch and modeling, this period is discretized into 20 time intervals of $15 \mathrm{~min}$. In each time interval, the loads and the output of the PVs are assumed to remain constant. To test the proposed approach in multiple scenarios, the network is divided into three districts, in which the load variability characteristics are different. The profiles of the PVs and the loads in the three districts are shown in Fig. 6 for the islanding period. "Time" in Fig. 6 refers to the start of the corresponding time interval.

The load weights are set as follows: the weights of the loads in nodes $7,10,11,13,16,22,28,43,45-48,59,60$, and 63 are set at 1 ; the weights of the loads in nodes 6,9 , $12,18,35,37,42,51,57$, and 62 are set at 5, and the weights of the other loads are set at 3 . These represent the relative priority of loads in the dispatch of supply.

The parameters for the PV/BE stations and the pluggedin EVs are shown in Tables 1,2 and 3. The rated charging/

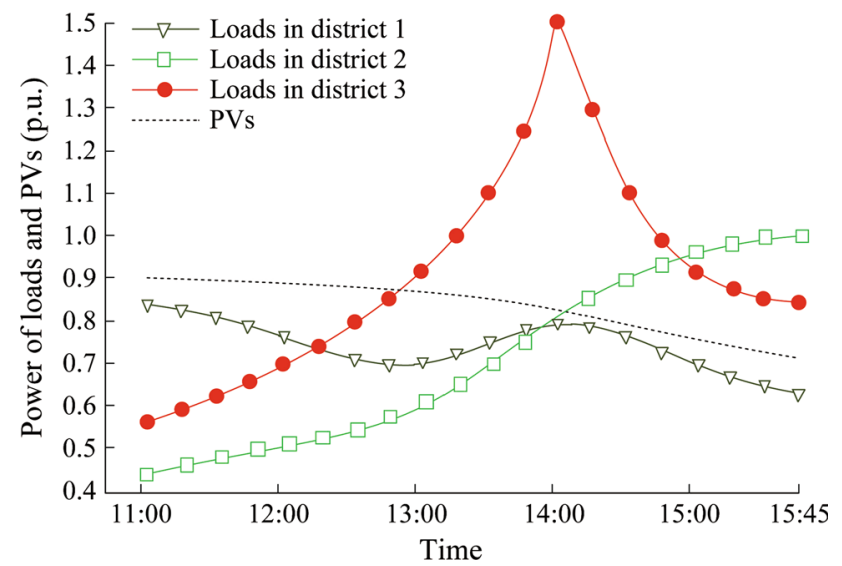

Fig. 6 Profiles of PVs and loads in the three districts 
Table 1 Parameters for PV stations

\begin{tabular}{ll}
\hline PV & Rated power $(\mathrm{kW})$ \\
\hline 1 & 100 \\
2 & 300 \\
3 & 100 \\
4 & 300 \\
5 & 100 \\
\hline
\end{tabular}

Table 2 Parameters for BE stations

\begin{tabular}{llll}
\hline BE & $\begin{array}{l}\text { BE energy } \\
\text { capacity }(\mathrm{kWh})\end{array}$ & $\begin{array}{l}\text { SOC of } \\
\text { BE at } T_{s}\end{array}$ & $\begin{array}{l}\text { BE maximum } \\
\text { charge/discharge } \\
\text { power }(\mathrm{kW})\end{array}$ \\
\hline 1 & 200 & 0.4 & 100 \\
2 & 300 & 0.7 & 150 \\
3 & 200 & 0.4 & 100 \\
4 & 300 & 0.5 & 100 \\
5 & 200 & 0.5 & 50 \\
\hline
\end{tabular}

Table 3 Parameters for EVs

\begin{tabular}{lll}
\hline EV & EV quantity & $\begin{array}{l}\text { SOC of E } \\
\text { integration at } T_{s}\end{array}$ \\
\hline 1 & 20 & 0.4 \\
2 & 20 & 0.8 \\
3 & 40 & 0.5 \\
4 & 15 & 0.4 \\
5 & 15 & 0.4 \\
\hline
\end{tabular}

discharging power through the $\mathrm{V} 2 \mathrm{G}$ facility of a single $\mathrm{EV}$ is $5 \mathrm{~kW}$. The capacity of a single EV is $15 \mathrm{kWh}$. The EV user participation rate $r_{\text {obey }}$ is set to be 0.6 .

\subsection{Island partition based on energy constraints}

The results for the first-stage island partition are shown in Fig. 7.

Nodes 4, 5 and 36 are converted into an equivalent node because the islands of PV 1 and PV 3 have an intersection, and Fig. 7 shows the final island partition result after node conversion.

\subsection{DER power dispatch and model modifications}

The dispatch test is applied to the four islands, and the results are shown in Table 4.

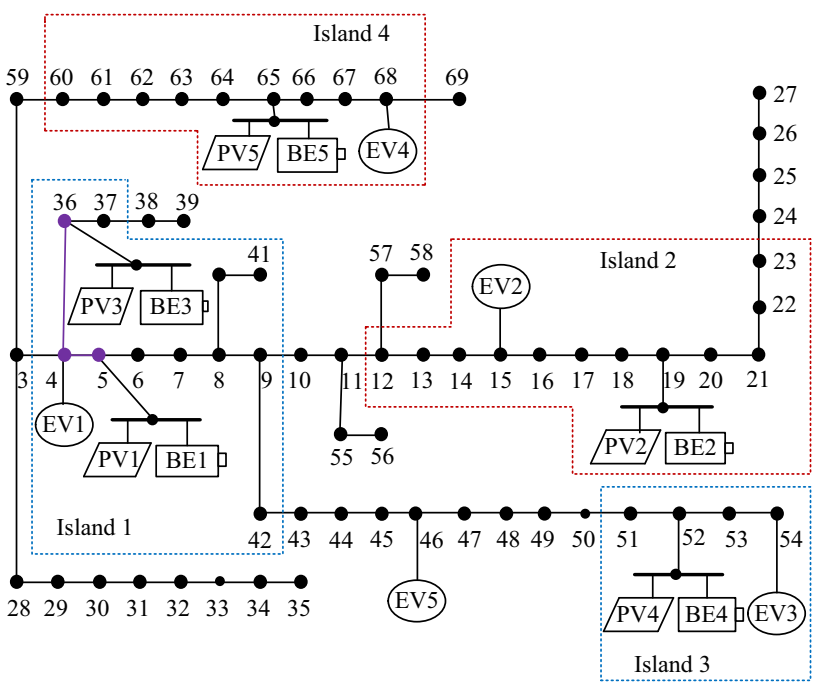

Fig. 7 Results for island partition model at first stage

Table 4 Dispatch results for the four islands

\begin{tabular}{llll}
\hline Island & $E_{\text {un }}(\mathrm{kWh})$ & $T_{\text {un }}$ & Result \\
\hline 1 & 0 & - & Passed \\
2 & 38.73 & - & Case B-2 \\
3 & 0 & - & Passed \\
4 & 10.36 & $2: 00-2: 15 \mathrm{PM}$ & Case A \\
\hline
\end{tabular}

According to Table 4, Islands 1 and 3 have passed the dispatch test, and Islands 2 and 4 have failed.

Islands 1 and 3 are in district 1 of the network, where the load varies smoothly. Considering the rated power of the PVs, the maximum charging/discharging powers and energy capacities of the BEs are sufficient to balance variability and transfer energy as needed.

For Island 4, the proposition in (23) is judged to be true and thus the situation belongs to Case A. Island 4 is in district 4 of the network, and according to Fig. 6, there are extreme moments during which the power demand is much higher than the average, while the maximum BE discharge power in Island 4 is relatively low. There is thus a time period (2:00-2:15 PM) during which the power demand cannot be met, even when the BEs and EVs discharge at maximum power. The dispatch results for Island 4 are shown in Table 5.

In Table 5, "Time" refers to the start of a time interval, the unit of BE power is $\mathrm{kW}$, and "SOC" represents the $\mathrm{SOC}$ of the $\mathrm{BE}$ at the end of the time interval. "EV" represents the number of EVs charging/discharging through the V2G facility, and is positive when discharging and negative when charging. In Table 5, the unsatisfied power demand $E_{u n}$ is concentrated around the time interval [13:30, 14:15], during which the loads are much higher 
Table 5 Dispatch results for Island 4

\begin{tabular}{|c|c|c|c|c|c|c|c|}
\hline Time & $\begin{array}{l}\text { BE5 power } \\
\text { (SOC) }\end{array}$ & EV4 & $P_{u n}$ & Time & $\begin{array}{l}\text { BE Power } \\
\text { (SOC) }\end{array}$ & EV4 & $P_{u n}$ \\
\hline 11:00 & $-26.1(53 \%)$ & 1 & 0 & $13: 30$ & $50.0(32 \%)$ & -3 & 25.5 \\
\hline $11: 15$ & $-31.7(57 \%)$ & 3 & 0 & $13: 45$ & $39.8(27 \%)$ & 8 & 0 \\
\hline $11: 30$ & $-2.34(58 \%)$ & -2 & 0 & 14:00 & $50.0(21 \%)$ & 7 & 13.6 \\
\hline $11: 45$ & $-7.92(58 \%)$ & 0 & 0 & $14: 15$ & $50.0(14 \%)$ & 6 & 2.32 \\
\hline $12: 00$ & $21.5(56 \%)$ & -5 & 0 & $14: 30$ & $36.2(10 \%)$ & 6 & 0 \\
\hline $12: 15$ & $3.00(55 \%)$ & 0 & 0 & $14: 45$ & $24.9(7 \%)$ & 5 & 0 \\
\hline $12: 30$ & $34.5(51 \%)$ & -5 & 0 & $15: 00$ & $8.58(6 \%)$ & 5 & 0 \\
\hline $12: 45$ & $36.1(47 \%)$ & -4 & 0 & $15: 15$ & $13.3(4 \%)$ & 4 & 0 \\
\hline 13:00 & $32.6(43 \%)$ & -2 & 0 & $15: 30$ & $18.0(2 \%)$ & 3 & 0 \\
\hline $13: 15$ & $36.6(38 \%)$ & 1 & 0 & $15: 45$ & $12.8(0 \%)$ & 4 & 0 \\
\hline
\end{tabular}

Table 6 Dispatch results for Island 2

\begin{tabular}{|c|c|c|c|c|c|c|c|}
\hline Time & $\begin{array}{l}\text { BE2 power } \\
\text { (SOC) }\end{array}$ & EV2 & $P_{u n}$ & Time & $\begin{array}{l}\text { BE2 Power } \\
\text { (SOC) }\end{array}$ & EV2 & $P_{u n}$ \\
\hline 11:00 & $-97.6(78 \%)$ & -2 & 0 & $13: 30$ & $22.1(92 \%)$ & 9 & 4.81 \\
\hline $11: 15$ & $-101(87 \%)$ & 3 & 0 & $13: 45$ & $75.4(86 \%)$ & 4 & 7.44 \\
\hline $11: 30$ & $-66.8(92 \%)$ & 0 & 0 & $14: 00$ & $84.6(79 \%)$ & 8 & 9.16 \\
\hline $11: 45$ & $-47.0(96 \%)$ & 0 & 0 & $14: 15$ & $94.4(71 \%)$ & 8 & 10.2 \\
\hline $12: 00$ & $-20.9(98 \%)$ & -2 & 0 & $14: 30$ & $117(61 \%)$ & 6 & 8.48 \\
\hline $12: 15$ & $-10.9(99 \%)$ & -2 & 0 & $14: 45$ & $133(50 \%)$ & 4 & 13.6 \\
\hline $12: 30$ & $-16.1(100 \%)$ & 1 & 0 & $15: 00$ & $129(39 \%)$ & 7 & 13.0 \\
\hline $12: 45$ & $6.41(99 \%)$ & -2 & 0.99 & $15: 15$ & $154(26 \%)$ & 7 & 8.28 \\
\hline 13:00 & $20.0(98 \%)$ & -2 & 0.09 & $15: 30$ & $164(13 \%)$ & 7 & 19.0 \\
\hline $13: 15$ & $48.3(94 \%)$ & -2 & 2.71 & $15: 45$ & $151(0 \%)$ & 6 & 57.1 \\
\hline
\end{tabular}

than average. The output of the BEs and EV integrations has reached the maximum discharge power during this period.

Island 2 is in district 2, where the variability trends of the loads have little consistency with that of the PVs, meaning that a high storage capacity is required to transfer the energy from the PVs.

The dispatch results for Island 2 are shown in Table 6 .

In Table 6 , the SOC of the $\mathrm{BE}$ is full during the period [12:30, 12:45]. After the calculation, energy waste is found to occur during the period [11:00, 12.45], so the situation of Island 2 belongs to Case B-2.

After adding the power constraint in (24) to the firststage island partition model for PV5/BE5 and adding the energy transfer constraints in (25)-(38) to the island partition model for PV2/BE2, the results for the modified firststage model are shown in Fig. 8.

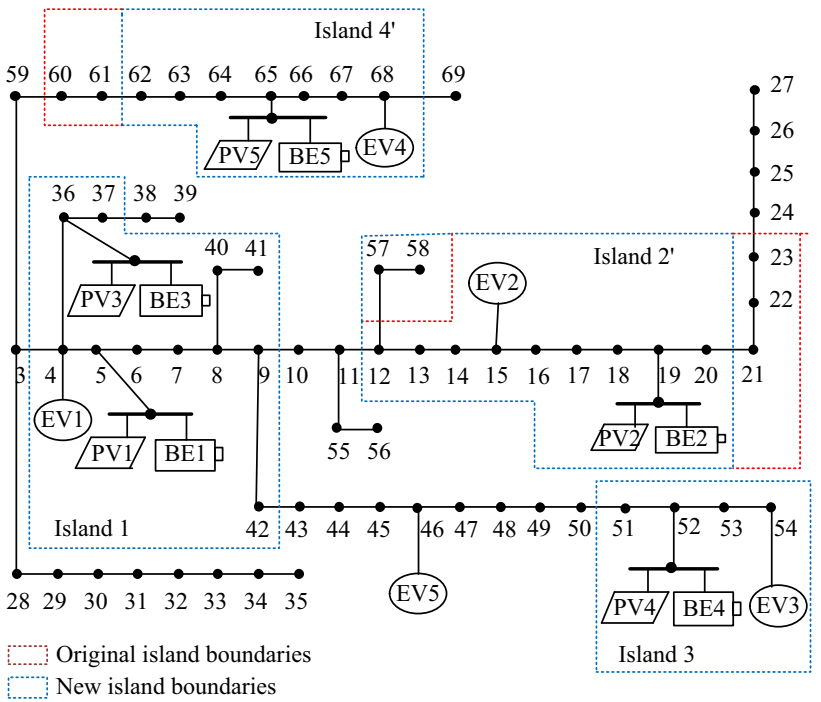

Fig. 8 Results for modified island partition model 
Table 7 Dispatch results for Island 1

\begin{tabular}{llll}
\hline Time & BE1 power (SOC) & BE3 power (SOC) & EV1 \\
\hline $11: 00$ & $27.3(47 \%)$ & $13.2(28 \%)$ & 3 \\
$11: 15$ & $25.5(43 \%)$ & $12.6(27 \%)$ & 3 \\
$11: 30$ & $23.7(40 \%)$ & $12.0(25 \%)$ & 3 \\
$11: 45$ & $21.6(38 \%)$ & $11.8(24 \%)$ & 3 \\
$12: 00$ & $23.0(35 \%)$ & $11.8(22 \%)$ & 2 \\
$12: 15$ & $19.2(32 \%)$ & $10.3(21 \%)$ & 2 \\
$12: 30$ & $13.7(31 \%)$ & $7.82(20 \%)$ & 3 \\
$12: 45$ & $10.2(29 \%)$ & $7.58(19 \%)$ & 3 \\
$13: 00$ & $3.60(29 \%)$ & $3.27(19 \%)$ & 3 \\
$13: 15$ & $8.98(28 \%)$ & $6.61(18 \%)$ & 3 \\
$13: 30$ & $17.4(26 \%)$ & $10.5(17 \%)$ & 3 \\
$13: 45$ & $26.3(22 \%)$ & $16.0(15 \%)$ & 2 \\
$14: 00$ & $31.5(19 \%)$ & $19.9(12 \%)$ & 2 \\
$14: 15$ & $29.2(15 \%)$ & $18.8(10 \%)$ & 2 \\
$14: 30$ & $24.2(12 \%)$ & $15.9(8 \%)$ & 2 \\
$14: 45$ & $22.3(9 \%)$ & $14.8(6 \%)$ & 2 \\
$15: 00$ & $20.5(6 \%)$ & $13.7(4 \%)$ & 2 \\
$15: 15$ & $18.4(4 \%)$ & $12.2(3 \%)$ & 2 \\
$15: 30$ & $16.7(2 \%)$ & $10.7(1 \%)$ & 2 \\
$15: 45$ & $14.2(0 \%)$ & $5.67(1 \%)$ & 2 \\
\hline & & &
\end{tabular}

Table 8 Dispatch results for Island 2'

\begin{tabular}{llllll}
\hline Time & $\begin{array}{l}\text { BE 2 power } \\
\text { (SOC) }\end{array}$ & EV2 & Time & $\begin{array}{l}\text { BE 2 power } \\
\text { (SOC) }\end{array}$ & EV2 \\
\hline $11: 00$ & $-46.1(74 \%)$ & 4 & $13: 30$ & $40.0(88 \%)$ & 2 \\
$11: 15$ & $-37.4(77 \%)$ & 3 & $13: 45$ & $64.6(83 \%)$ & 3 \\
$11: 30$ & $-44.0(81 \%)$ & 3 & $14: 00$ & $88.3(76 \%)$ & 3 \\
$11: 45$ & $-40.1(84 \%)$ & 3 & $14: 15$ & $96.2(68 \%)$ & 3 \\
$12: 00$ & $-31.7(87 \%)$ & 2 & $14: 30$ & $104(59 \%)$ & 4 \\
$12: 15$ & $-27.4(89 \%)$ & 2 & $14: 45$ & $112(50 \%)$ & 4 \\
$12: 30$ & $-22.1(91 \%)$ & 2 & $15: 00$ & $119(40 \%)$ & 4 \\
$12: 45$ & $-16.0(92 \%)$ & 2 & $15: 15$ & $133(29 \%)$ & 5 \\
$13: 00$ & $-9.07(93 \%)$ & 2 & $15: 30$ & $146(16 \%)$ & 5 \\
$13: 15$ & $15.3(92 \%)$ & 2 & $15: 45$ & $162(3 \%)$ & 6 \\
\hline
\end{tabular}

After the modification, nodes 60 and 61 are excluded from Island 4 and nodes 21-23 are excluded from Island 2. Nodes 57 and 58 are included in Island 2'.

The dispatch results for Islands 1, 2', 3 and 4' are shown in Tables 7, 8, 9 and 10. $E_{u n}$ is 0 for each of these islands. All of the PVs operate under MPPT conditions.

According to the schedule of Island 2' (Table 8), the BE charges during the period [11:00, 13:15] and discharges during the period $[13: 15,16: 00]$ to transfer the energy of the PV, and the EV integration's output power is also relatively high during the period [14:30, 16:00].
Table 9 Dispatch results for Island 3

\begin{tabular}{llllll}
\hline Time & $\begin{array}{l}\text { BE 4 power } \\
\text { (SOC) }\end{array}$ & EV3 & Time & $\begin{array}{l}\text { BE 4 Power } \\
\text { (SOC) }\end{array}$ & EV3 \\
\hline $11: 00$ & $31.1(47 \%)$ & 6 & $13: 30$ & $28.4(25 \%)$ & 4 \\
$11: 15$ & $31.1(45 \%)$ & 5 & $13: 45$ & $32.0(22 \%)$ & 5 \\
$11: 30$ & $30.4(42 \%)$ & 5 & $14: 00$ & $35.6(19 \%)$ & 6 \\
$11: 45$ & $29.6(40 \%)$ & 5 & $14: 15$ & $35.1(17 \%)$ & 6 \\
$12: 00$ & $28.9(37 \%)$ & 4 & $14: 30$ & $34.5(14 \%)$ & 6 \\
$12: 15$ & $26.8(35 \%)$ & 4 & $14: 45$ & $34.1(11 \%)$ & 6 \\
$12: 30$ & $24.8(33 \%)$ & 3 & $15: 00$ & $33.6(8 \%)$ & 5 \\
$12: 45$ & $22.7(31 \%)$ & 2 & $15: 15$ & $32.8(5 \%)$ & 5 \\
$13: 00$ & $20.6(30 \%)$ & 1 & $15: 30$ & $32.0(3 \%)$ & 4 \\
$13: 15$ & $24.6(27 \%)$ & 3 & $15: 45$ & $31.3(0 \%)$ & 4 \\
\hline
\end{tabular}

In Island 4' (Table 10), because the number of dischargeable EVs is conservatively estimated based on the total energy stored in the EV integration in (16), the EV integration initially charge during the period [11:00, 12:30] to ensure sufficient energy will be available during the period [13:30, 14:30], when the loads are much higher than average.

Based on the simulation results, the BEs are important and necessary to relieve the imbalance between the PVs and the loads and transfer the energy from the PVs. By contrast, EV integrations are less controllable considering the EV user participation and the conservative estimation of the number of chargeable/dischargeable EVs.

\subsection{Power flow simulation}

Sequential power flow is performed based on the dispatch results. Nodes 5, 19, 52 and 65 are the slack nodes of the 4 islands. The result shows that the power loss can be covered by the slack PV generation, and the voltage deviation is very small. The voltage profiles and power losses of the 4 islands under peak load conditions (Island 1 and 3 at 11:00, Island 2 at 15:45, Island 4 at 14:00) are shown in Fig. 9.

\subsection{Comparison with previous methods}

For comparison, the island partition methods proposed by $[11,19]$ are implemented for the same distribution network.

Since the variability of loads and the dynamic dispatch of DERs are not considered in these previous studies (the static power dispatch of DERs in [19] is independent of the island partition process, and is done when the island scope has been determined), the static values of loads and DERs are defined as follows to ensure the feasibility of the islands: 
Table 10 Dispatch results for Island 4'

\begin{tabular}{|c|c|c|c|c|c|}
\hline Time & BE5 power (SOC) & EV4 & Time & BE 5 power (SOC) & EV1 \\
\hline 11:00 & $12.3(48 \%)$ & -3 & $13: 30$ & $25.2(27 \%)$ & 4 \\
\hline $11: 15$ & $12.9(47 \%)$ & -3 & $13: 45$ & $29.6(24 \%)$ & 5 \\
\hline $11: 30$ & $13.4(45 \%)$ & -2 & $14: 00$ & $36.0(19 \%)$ & 6 \\
\hline $11: 45$ & $13.9(43 \%)$ & -2 & $14: 15$ & $30.7(15 \%)$ & 5 \\
\hline $12: 00$ & $14.3(42 \%)$ & -1 & $14: 30$ & $25.7(12 \%)$ & 4 \\
\hline $12: 15$ & $15.3(40 \%)$ & -1 & $14: 45$ & $20.9(10 \%)$ & 4 \\
\hline $12: 30$ & $16.3(38 \%)$ & 0 & 15:00 & $16.4(8 \%)$ & 3 \\
\hline $12: 45$ & $17.2(36 \%)$ & 0 & $15: 15$ & $16.0(6 \%)$ & 3 \\
\hline 13:00 & $18.1(33 \%)$ & 0 & $15: 30$ & $15.7(4 \%)$ & 3 \\
\hline $13: 15$ & $21.6(31 \%)$ & 2 & $15: 45$ & $15.4(2 \%)$ & 3 \\
\hline
\end{tabular}

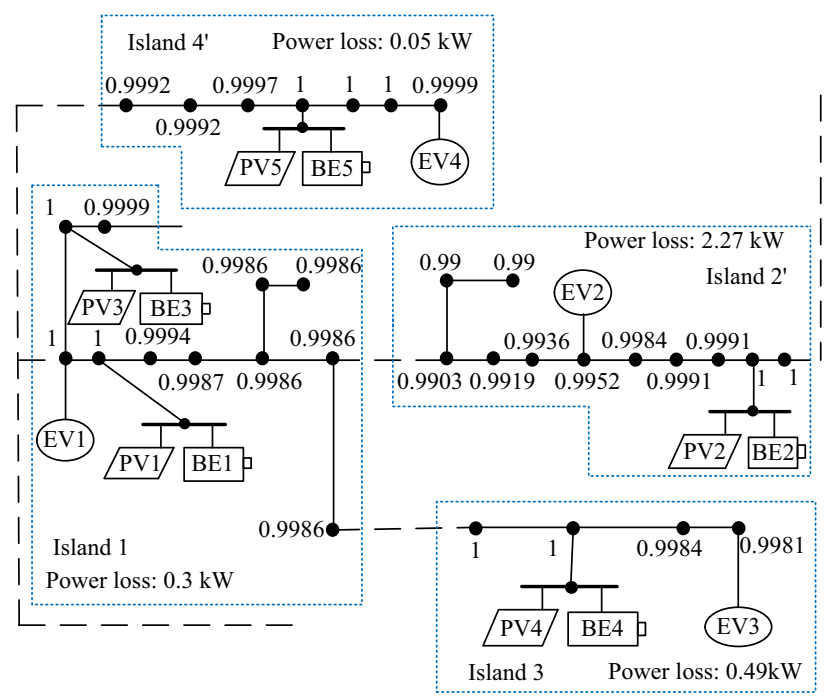

Fig. 9 Voltage profiles and power losses

1) The BEs and EV integrations discharge completely by the end of the islanding period, and the discharging power is considered to be constant.

2) The PV generators are controlled to operate with MPPT, but their static values are set to their minimum power output during the entire islanding period.

3) The loads are set to their peak value during the islanding period.

The same island partition is obtained by the methods in [11, 19], and is compared with the result obtained by proposed method in Fig. 10.

The total restored load (weighted value) by the method in [11] (or [19]) and the proposed method is $2484 \mathrm{~kW}$ and $3516 \mathrm{~kW}$ respectively, and the total restored energy (weighted value) during the islanding period is $9165 \mathrm{kWh}$ and $12935 \mathrm{kWh}$ respectively.

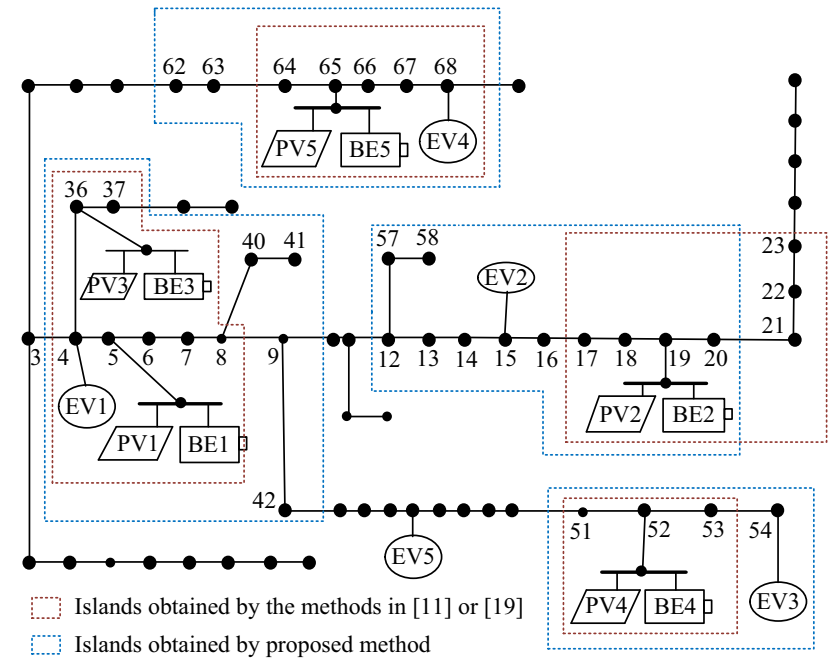

Fig. 10 Comparison of the island partition results

The computational complexity of the methods in $[11,19]$ and this paper are all no more than $O\left(N^{2}\right)$. The methods are programmed in the MATLAB environment [21]. The YALMIP toolbox is applied for methods in [11] and proposed method. The simulations have been run on a desktop PC containing an Intel ${ }^{\circledR}$ Core $^{\mathrm{TM}}$ i7-2600 3.40 $\mathrm{GHz}$ CPU, and $16 \mathrm{~GB}$ of RAM. The computing time of the methods in $[11,19]$ and this paper are $1.5 \mathrm{~s}, 4.0 \mathrm{~s}$ (only island partition considered) and $15.1 \mathrm{~s}$ respectively. The proposed method needs more computing time because of the DER dispatch and model modification processes.

\section{Conclusion}

This paper has presented an integrated islanding approach for an ADN when a failure occurs, in which an initial island partition is determined in the first stage and 
then refined through two steps of tests in the second stage.

An energy index is introduced to the island partition, and an innovative energy-constrained optimal island partition model is proposed. Previous works formulated a static optimal island partition problem as a tree knapsack problem (TKP) based on the assumption that the loads and the outputs of the DGs are constant. The introduction of the energy index and the energy constraints allow the island partition model to remain in the TKP form and thus be solvable, meanwhile taking the variability of the loads and the DGs into consideration.

In the second stage, the DER power dispatch process and sequential power flow are performed to test the islands because the energy constraint is not a sufficient condition. Modifications of the island partition model based on the dispatch results and adjustment strategies based on the power flow are also provided. The strengths of the energy constraint, the energy and power constraint and the energy transfer constraint are incremented to adapt to different situations.

Controllable energy storage is essential for both power balancing and frequency stabilization in island mode operation. It may be provided by battery systems, in this paper assumed to be integrated with PV generation, or by plugged-in electric vehicles when their owners have allowed charge/discharge control for network support. The maximum charge/discharge power and energy capacity are the determining factors of the ability to provide power support and energy transfer using energy storage equipment. The load and DG variability characteristics also affect the energy storage requirements in the island mode operation.

The proposed method needs more computing time than previous methods for static load and generation, because of the DER dynamic dispatch and model modification processes, but the load restoration performance of proposed method is much better than that of the conventional methods. The proposed method prevents curtailment of $42 \%$ more load and delivers $41 \%$ more energy than the previous methods.

Acknowledegements This work was supported in part by the National Science Foundation of China (No. 51477029), the National Science and Technology Support Program of China (No. 2015BAA01B01) and the State Grid Corporation of China (No. SGTYHT/14-JS-188).

Open Access This article is distributed under the terms of the Creative Commons Attribution 4.0 International License (http:// creativecommons.org/licenses/by/4.0/), which permits unrestricted use, distribution, and reproduction in any medium, provided you give appropriate credit to the original author(s) and the source, provide a link to the Creative Commons license, and indicate if changes were made.

\section{References}

[1] D'Adamo C, Abbey C, Jupe S (2011) Development and operation of active distribution networks: results of CIGRE C6. 11 working group. In: Proceedings of the CIRED 21st international conference on electricity distribution, Frankfurt, Germany, Jun 2011, pp 6-9

[2] Conti S, Rizzo SA (2015) Monte Carlo simulation by using a systematic approach to assess distribution system reliability considering intentional islanding. IEEE Trans Power Delivery 30(1):64-73

[3] Agarwal L, Wang P, Goel L (2014) Using EV battery packs for vehicle-to-grid applications: an economic analysis. In: Proceedings of the IEEE 2014 innovative smart grid technologiesAsia, Kuala Lumpur, Malaysia, 20-23 May 2014, pp 663-668

[4] Al-Muhaini M, Heydt GT (2013) Evaluating future power distribution system reliability including distributed generation. IEEE Trans Power Delivery 28(4):2264-2272

[5] Caldon R, Stocco A, Turri R (2008) Feasible of adaptive intentional islanding operation of electric utility systems with distributed generation. Electr Power Syst Res 78(12):2017-2023

[6] Mehrasa M, Pouresmaeil E, Jørgensen BN et al (2015) A control plan for the stable operation of microgrids during grid-connected and islanded modes. Electr Power Syst Res 129:10-22

[7] Jayaweera D, Galloway S, Burt G et al (2013) A sampling approach for intentional islanding of distributed generation. IEEE Trans Power Syst 28(2):514-521

[8] Arefifar S, Mohamed YR, EL-Fouly T (2013) Optimum microgrid design for enhancing reliability and supply-security. IEEE Trans Smart Grid 4(3):1567-1575

[9] Mao Y, Miu KN (2003) Switch placement to improve system reliability for radial distribution systems with distributed generation. IEEE Trans Power Syst 18(4):1346-1352

[10] Li J, Liu CC, Schneider KP (2010) Controlled partitioning of a power network considering real and reactive power balance. IEEE Trans Smart Grid 1(3):261-269

[11] Ling J, Wang X, Wu P et al (2012) Two-stage method for optimal island partition of distribution system with distributed generations. IET Gener Transm Distrib 6(3):218-225

[12] Lu Y, Yi X, Wu J et al (2006) An intelligent islanding technique considering load balance for distribution system with DGs. In: Proceedings of the 2006 IEEE power and energy society general meeting, Montreal, Que, Canada, Jun 2006, pp 5-9

[13] Ding L, Pan Z, Cong W et al (2008) Rooted tree based searching strategies for intentional islanding of distributed generation. In: Proceedings of the 2008 IET 9th international conference on developments in power system protection, Glasgow, UK, 17-20 March 2008, pp 302-307

[14] Oboudi MH, Hooshmand R, Karamad A (2015) Feasible method for making controlled intentional islanding of microgrids based on the modified shuffled frog leap algorithm. Int J Electr Power Energy Syst 78:745-754

[15] Lee SH, Son G, Park JW (2013) Power management and control for grid-connected DGs with intentional islanding operation of inverter. IEEE Trans Power Syst 28(2):1235-1244

[16] Gouveia C, Moreira J, Moreira CL et al (2013) Coordinating storage and demand response for microgrid emergency operation. IEEE Trans Smart Grid 4(4):1898-1908

[17] Khodaei A (2014) Microgrid optimal scheduling with multiperiod islanding constraints. IEEE Trans Power Syst 29(3):1383-1392

[18] Ahn SJ, Nam SR, Choi JH et al (2013) Power scheduling of distributed generators for economic and stable operation of a microgrid. IEEE Trans Smart Grid 4(1):398-405 
[19] Zhang M, Chen J (2015) Islanding and scheduling of power distribution systems with distributed generation. IEEE Trans Power Syst 30(6):3120-3129

[20] Adhikari S, Li F (2014) Coordinated V-f and P-Q control of solar photovoltaic generators with MPPT and battery storage in microgrids. IEEE Trans Smart Grid 5(4):1270-1281

[21] Löfberg J (2004) Yalmip: a toolbox for modeling and optimization in MATLAB. In: Proceedings of the 2004 IEEE international symposium on computer aided control systems design, Taipei, Taiwan, China,2-4 September, pp 284-289

Junpeng ZHU received his B.Sc. degree in Mathematics from Southeast University, China, in 2012. He is currently pursuing a Ph.D. degree in Electrical Engineering at Southeast University. His research interests include intentional island partition and network reconfiguration in active distribution networks.

Wei GU received his B.Eng. and Ph.D. degrees in Electrical Engineering from Southeast University, China, in 2001 and 2006, respectively. From 2009 to 2010, he was a Visiting Scholar in the Department of Electrical Engineering, Arizona State University, Tempe, AZ, USA. He is now a professor in the School of Electrical Engineering at Southeast University. His research interests include distributed generation and microgrids, active distribution networks, and power quality.
Ping JIANG received his B.Eng. and Ph.D. degrees from Southeast University, Nanjing, China in 1982 and 2010 respectively. He is now a professor in the School of Electrical Engineering, Southeast University. His research interests are power system operation and control, power electronic and its applications in power systems.

Zhi WU received a B.Sc. degree in Mathematics from Southeast University, China, in 2009, and an M.Eng. degree in Electrical Engineering from the School of Electrical Engineering, Southeast University 2012, and a Ph.D. degree in Electrical Engineering from the School of Electronic, Electrical and Systems Engineering, University of Birmingham, U.K. His research interests include renewable energy, smart grid energy management systems, the electricity market, and optimization techniques.

Xiaodong YUAN received his B.Eng. and M.Eng. degrees in Electrical Engineering from Southeast University, China, in 2001 and 2004, respectively. He is now a senior engineer in Jiangsu Electric Power Company Research Institute. His research interests include renewable energy and power quality.

Yinghui NIE received her B.Eng. degree in Electrical Engineering from Southeast University, China, in 2014. She is currently pursuing an M.Eng. degree in Electrical Engineering at Southeast University. Her research interests include intentional island partition and reliability assessment in active distribution networks. 